\title{
Anisotropy-driven thermal conductivity switching and thermal hysteresis in a ferroelectric
}

Cite as: Appl. Phys. Lett. 115, 192903 (2019); https://doi.org/10.1063/1.5127825

Submitted: 13 September 2019 . Accepted: 27 October 2019 . Published Online: 07 November 2019

Juan Antonio Seijas-Bellido, Jorge Î́ñiguez (D), and Riccardo Rurali (D)

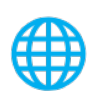

View Online

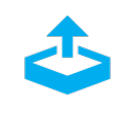

Export Citation

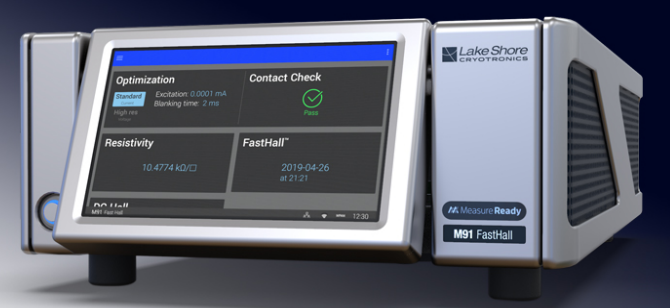

Mn Measure Ready M91 FastHall ${ }^{\text {TM }}$ Controller

A revolutionary new instrument for complete Hall analysis

See the video 8 


\title{
Anisotropy-driven thermal conductivity switching and thermal hysteresis in a ferroelectric
}

Cite as: Appl. Phys. Lett. 115, 192903 (2019); doi: 10.1063/1.5127825

Submitted: 13 September 2019 . Accepted: 27 October 2019 .

Published Online: 7 November 2019

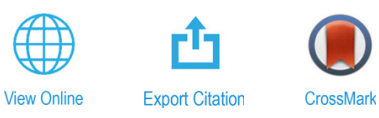

Juan Antonio Seijas-Bellido, Jorge Íñiguez, ${ }^{2,3}$ (D) and Riccardo Rurali ${ }^{1, a)}$ (iD

\section{AFFILIATIONS}

'Institut de Ciència de Materials de Barcelona (ICMAB-CSIC), Campus de Bellaterra, 08193 Bellaterra, Barcelona, Spain

${ }^{2}$ Materials Research and Technology Department, Luxembourg Institute of Science and Technology (LIST),

Avenue des Hauts-Fourneaux 5, L-4362 Esch/Alzette, Luxembourg

${ }^{3}$ Physics and Materials Science Research Unit, University of Luxembourg, 41 Rue du Brill, L-4422 Belvaux, Luxembourg

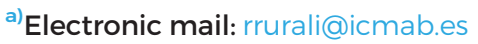

\begin{abstract}
We present a theoretical proposal for the design of a thermal switch based on the anisotropy of the thermal conductivity of $\mathrm{PbTiO}_{3}$ and the possibility to rotate the ferroelectric polarization with an external electric field. Our calculations are based on an iterative solution of the phonon Boltzmann Transport Equation and rely on interatomic force constants computed within an efficient second-principles density functional theory scheme. We also characterize the hysteresis cycle of the thermal conductivity in the presence of an applied electric field and show that the response time would be limited by the speed of the ferroelectric switch itself and thus the switch can operate in the highfrequency regime.
\end{abstract}

Published under license by AIP Publishing. https://doi.org/10.1063/1.5127825

A long-standing goal of "phononics"1-3 — the discipline that studies phonon manipulation-is the implementation of a phonon-based Boolean algebra, where operations rely on a high and a low conductance state, which are used to encode the logical values of 1 and 0 . A fundamental requisite to perform logic operations is having access to two conductance states that are as different as possible and being able to commute back and forth between them.

Many materials are anisotropic and thus provide natural access to such distinct conductance states, but practical implementations are nevertheless hindered. On the one hand, the anisotropy is often small, like in wurtzite crystals, ${ }^{4-6}$ where $\kappa_{x x} / \kappa_{y y}$ may range from $0.96(\mathrm{GaP})$ to $1.18(\mathrm{ZnSe})$ at $300 \mathrm{~K}$. In other materials, however, it can be much larger and a convenient limiting case is graphite: in that case, the inplane thermal conductivity is four orders of magnitude larger than the out-of-plane one, ${ }^{7}$ along which phonon propagation is mediated by weak van der Waals forces. On the other hand, and more importantly, switching from one conductance state to the other is normally not possible, because the device design determines the element of the thermal conductivity tensor that is relevant for phonon transport (i.e., heat flows along a given crystallographic direction, and the sample cannot be rotated).

Ferroelectric perovskite oxides are anisotropic materials whose anisotropy is determined by the off-center displacement of the cations with respect to the surrounding oxygen cages. This distortion of the lattice has an associated polarization that can thus be reoriented or fully reversed with an external electric field. This property allows designing materials with tunable thermal properties by the continuous control of the distortion of the lattice ${ }^{8,9}$ or by "writing/erasing" domain walls that separate contiguous domains with differently oriented polarizations. ${ }^{10,11}$ In this paper, we discuss an even simpler effect, namely, how an electric field can be used to rotate the polarization in a monodomain and, consequently, gain access to a different element of the thermal conductivity tensor within a given device setup, thus implementing a thermal switch. As we discuss below, this approach does not require the design of complex multidomains and only relies on the anisotropy of the thermal conductivity in the monodomain state.

We study $\mathrm{PbTiO}_{3}$, a paradigmatic oxide with a perovskite structure that is ferroelectric below $760 \mathrm{~K}$. We calculate its ground state structure, the harmonic and third-order anharmonic interatomic force constants (IFCs) within second-principles density-functional theory (SPDFT) as implemented in the SCALE-UP code. ${ }^{12,13}$ SPDFT reproduces the vibrational and response properties of $\mathrm{PbTiO}_{3}{ }^{12}$ with an accuracy comparable to most first-principles approaches, and it has a documented predictive power for the most important structural, vibrational, and response properties of ferroelectric perovskite oxides. ${ }^{14,15}$ 
In particular, the second-principles model potential of $\mathrm{PbTiO}_{3}$ used in this work ${ }^{12}$ has been repeatedly shown to reproduce the experimental behavior of the material with good accuracy, except for a significant underestimate of the ferroelectric transition temperature (the model predicts $510 \mathrm{~K}$ ), not relevant for our present purposes. Regarding the thermal conductivity, a central quantity for the purpose of this manuscript, a meaningful comparison with the experiments is not possible. The only experimental measurements reported to date were carried out in multidomain samples, and thus, (i) the measured $\kappa$ can be expected to be much lower than that in a monodomain sample $\mathrm{e}^{10,11,16,17}$ and (ii) the anisotropy cannot be estimated. Nevertheless, the good agreement of our computed harmonic properties, i.e., the phonon dispersion, with density-functional theory (DFT) ${ }^{12,13}$ and experiments, ${ }^{14,15}$ suggests that our results are reliable. The IFCs are then used as an input to solve iteratively the Boltzmann Transport Equation (BTE) with the ShengBTE code, ${ }^{18}$ and the lattice thermal conductivity is obtained as

$$
\kappa_{i j}=\sum_{\lambda} \kappa_{i j, \lambda}=C \sum_{\lambda} f_{\lambda}\left(f_{\lambda}+1\right)\left(h \nu_{\lambda}\right)^{2} v_{i, \lambda} F_{j, \lambda},
$$

where $i$ and $j$ are the spatial directions $x, y$, and $z . C^{-1}=k_{B} T^{2} \Omega N$, where $k_{B}, h, T, \Omega$, and $N$ are Boltzmann's constant, Planck's constant, the temperature, the volume of the 5-atom unit cell, and the number of q-points, respectively. The sum runs over all phonon modes, with the index $\lambda$ including both the $\mathbf{q}$-point and the phonon band. $f_{\lambda}$ is the equilibrium Bose-Einstein distribution function, and $\nu_{\lambda}$ and $v_{i, \lambda}$ are the frequency and group velocity of phonon $\lambda$, respectively. The mean free displacement $F_{j, \lambda}$ is initially taken to be equal to $\tau_{\lambda} v_{j, \lambda}$, where $\tau_{\lambda}$ is the lifetime of mode $\lambda$ within the relaxation time approximation (RTA). Starting from this guess, the solution is then obtained iteratively and $F_{j, \lambda}$ takes the general form $\tau_{\lambda}\left(v_{j, \lambda}+\Delta_{j, \lambda}\right)$, where the correction $\Delta_{\lambda}$ captures the changes in the heat current associated with the deviations in the phonon populations computed at the RTA level. ${ }^{19,20}$ Scattering from isotopic disorder is also included considering the natural isotopic distributions of $\mathrm{Pb}, \mathrm{Ti}$, and $\mathrm{O}$, through the model due to Tamura. $^{21}$

The thermal conductivity tensor of $\mathrm{PbTiO}_{3}$ in the ferroelectric ground state has two independent components and has the form

$$
\boldsymbol{\kappa}=\left[\begin{array}{ccc}
\kappa^{\perp} & 0 & 0 \\
0 & \kappa^{\perp} & 0 \\
0 & 0 & \kappa^{\|}
\end{array}\right],
$$

where $\kappa^{\perp}$ and $\kappa^{\|}$are the thermal conductivities that account for heat transport when phonons flow perpendicular or parallel to the polarization, $\mathbf{P}$. The computed values of the thermal conductivity are plotted in Fig. 1, where it is easy to see that $\kappa^{\perp}>\kappa^{\|}$throughout the temperature range investigated. The anisotropy, $\kappa^{\perp} / \kappa^{\|}$, is larger at low temperatures (with a peak value of $\sim 4$ at $100 \mathrm{~K}$ ), as shown in the inset of Fig. 1, but at room temperature, it is still larger than 2. This anisotropy can be used to implement a thermal switch, as schematically illustrated in Fig. 2: phonons that propagate along the $x$-axis will experience a large thermal conductivity, $\kappa^{\perp}$, when $\mathbf{P}$ is oriented along $y$ or $z$, while the conductivity will be lower than and equal to $\kappa^{\|}$when $\mathbf{P}$ is parallel to the heat transport direction, $x$. Commutation between the two conductance states is achieved with a rotation of the polarization (which is equivalent to a rotation of the lattice/sample) by means of an electric field that acts as the gate control signal.

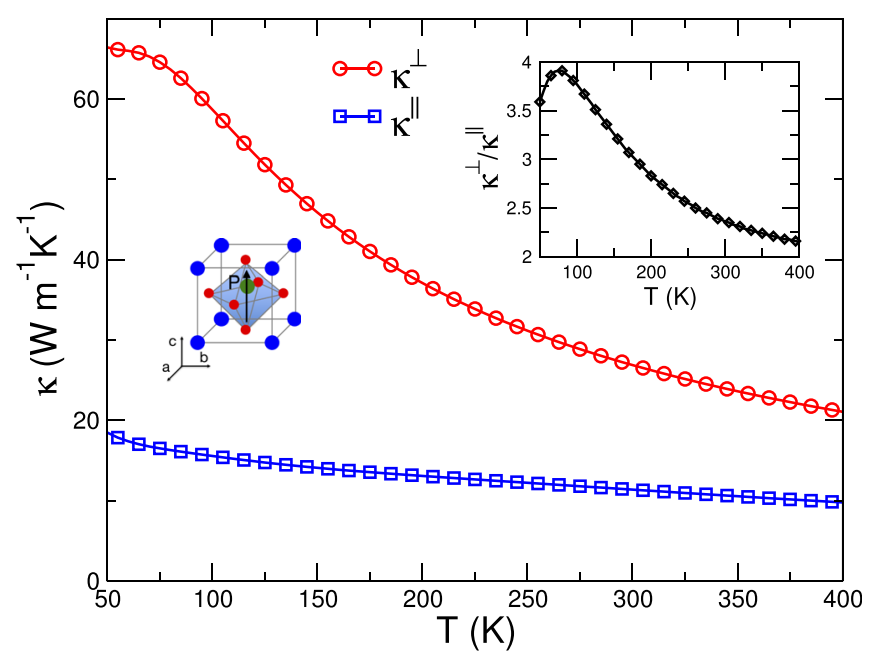

FIG. 1. Thermal conductivity as a function of temperature of $\mathrm{PbTiO}_{3}$ in the ferroelectric ground state along the $c$-axis and parallel to the polarization, $\kappa^{\|}$, and in the ab-plane and perpendicular to the polarization, $\kappa^{\perp}$. The ratio $\kappa^{\perp} / \kappa^{\|}$, which quantifies the thermal anisotropy of $\mathrm{PbTiO}_{3}$, is shown in the inset.

We now take a closer look at the way the thermal conductivity, $\boldsymbol{\kappa}$, switches between the low and the high state. To this end, we vary adiabatically an electric field, $\mathrm{E}$, perpendicular to the polarization to rotate the latter back and forth between two equivalent ground states of the $\mathrm{PbTiO}_{3}$ lattice. In the limit of very slow variations of $\mathbf{E}$, we can assume

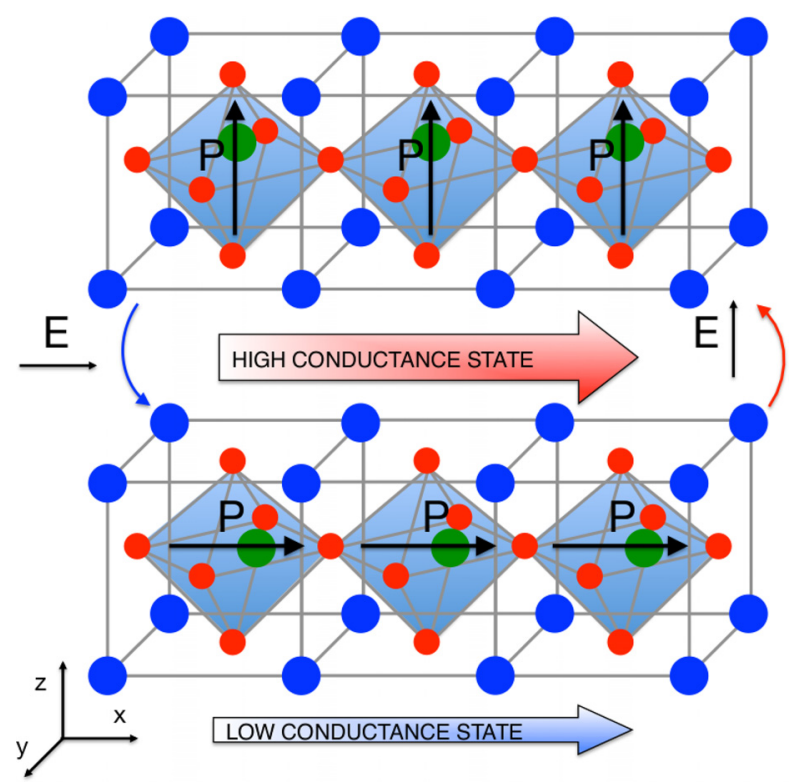

FIG. 2. Sketch of the thermal switch. In the high conductance state, phonons flow along a direction perpendicular to $\mathbf{P}$ and the thermal conductivity is $\kappa^{\perp}$. In the low conductance state, phonons flow parallel to $\mathbf{P}$ and the thermal conductivity is $\kappa^{\|}$. An electric field along the $x$-axis rotates $\mathbf{P}$ and triggers the commutation from highto-low conductivity, while an electric field along the z-axis (or, equivalently, along the $y$-axis) triggers the low-to-high conductivity transition. 
to be always in equilibrium, and thus, we can define and calculate the thermal conductivity. Notice that we assume this adiabatic approximation only to study the evolution of the thermal conductivity with the electric field, i.e., with the rotation of the polarization, and discuss its hysteretic response. This is a not a requisite for the operation of the switch, and, of course, in an application, one would like to commute between conductance states as fast as possible. We discuss this situation below.

We take as the initial state the one with the polarization parallel to the phonon flow, that is, the low conductivity state. Then, we start to apply a perpendicular electric field of increasing strength: $\mathbf{P}$ starts precessing and the conductivity is reduced (curve la in Fig. 3); this is a result of the field-induced lowering of the lattice symmetry, which increases the phase-space for three-phonon scattering events and thus reduces their lifetimes. ${ }^{8}$ When the coercive field, $E_{c o e}$, is reached, the polarization switches (curve $1 \mathrm{~b}$ in Fig. 3) and becomes parallel to the electric field. If now the electric field is reduced until it vanishes (curve 2 in Fig. 3), a ground state equivalent to the starting configuration is reached. Now, however, $\mathbf{P}$ is perpendicular to the direction of phonon propagation and the system is in the high conductivity state. To rotate back the polarization, we start applying an electric field along $x$, the direction of the heat flow: like before, $\boldsymbol{\kappa}$ decreases until $\mathbf{P}$ switches (curves $3 \mathrm{a}$ and $3 \mathrm{~b}$ in Fig. 3). Finally, upon removal of the electric field (curve 4 in Fig. 3), we recover the starting configuration. As it can be seen, $\boldsymbol{\kappa}$ follows a hysteresis cycle and it can take different values at a given electric field, depending on how the field changed in the past. This is a direct consequence of the fact that given a value of $\mathbf{E}$ one cannot univocally know $\mathbf{P}$, which ultimately determines the thermal conductivity along a given direction.

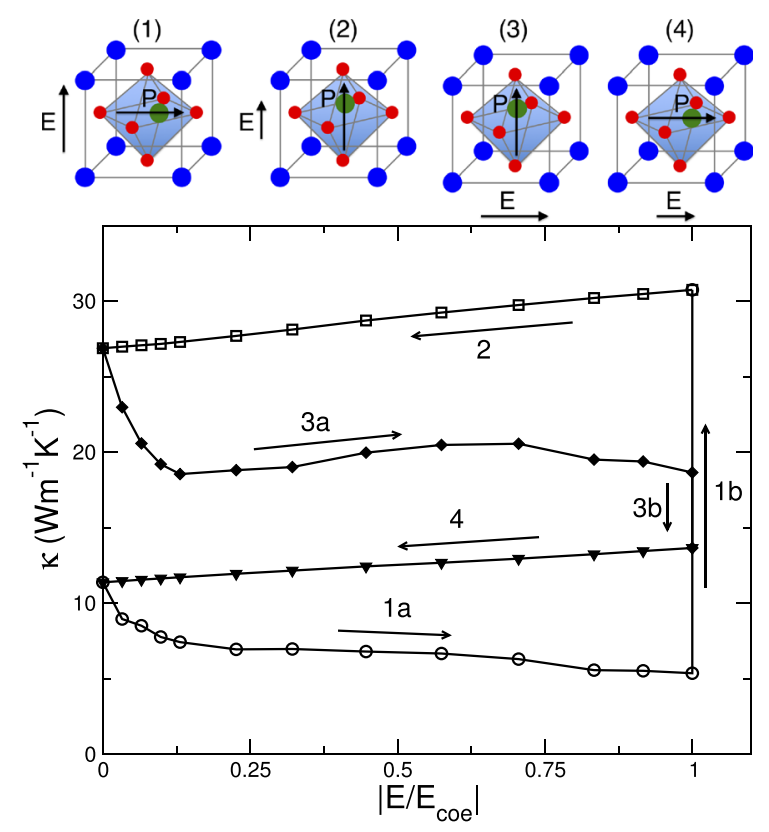

FIG. 3. Hysteresis cycle followed by the thermal conductivity, $\boldsymbol{\kappa}$, when the polarization is rotated back and forth at $300 \mathrm{~K}$. The sketches in the upper part of the figure depict the direction of the electric field (polarization), throughout (at the beginning of) the corresponding branch of the $\boldsymbol{\kappa}(\mathbf{E})$ curve; long (short) arrows indicate the increasing (decreasing) values of $\mathbf{E}$.
The relation between $\mathbf{E}, \mathbf{P}$, and $\boldsymbol{\kappa}$ is more clearly illustrated in Fig. 4 where one full hysteresis cycle is displayed as a function of time, which is assumed to vary conveniently slow to allow us to consider the transition adiabatic and the system in instantaneous equilibrium. Like in Fig. 3, $\mathbf{P}$ is initially taken to lie along the $x$-axis so that the system is in the low conductance state and an electric field $E_{z}$ must be applied to rotate the polarization and switch to the high conductance state. This plot highlights the role of temperature in determining the difference between the two conductance states, which is much larger at lower temperature, as already shown in the inset of Fig. 1.

In the above discussion, the electric field was varied with slow ramps so that the transition could be considered adiabatic, the system was always in equilibrium, and $\boldsymbol{\kappa}$ could be computed all along the hysteresis cycle. Of course, one would like to commute between 1 and 0 , i.e., high- and low-conductances, as quick as possible and thus would rather use short electric field pulses to rotate the polarization. Although $\boldsymbol{\kappa}$ cannot be calculated in these (strongly) out-of-equilibrium conditions, we can infer on the overall relaxation time of the low-tohigh and high-to-low commutation, which is important information to estimate the maximum operation frequency of the thermal switch. In general, if at a given time, all the modes propagate along, say, $x$ under a certain lattice potential, and then something happens (change in potential, removal of the temperature gradient, etc.); such a propagation will continue for a time that will depend on the phonon lifetimes. More precisely, we introduce an effective relaxation time as

$$
\tau^{\perp, \|}=\frac{1}{\kappa^{\perp, \|}} \sum_{\lambda} \tau_{\lambda} \kappa_{\lambda}^{\perp, \|}
$$

where the relaxation time of each mode $\tau_{\lambda}$ is weighted with its contribution to the thermal conductivity, $\kappa_{\lambda}^{\perp} / \kappa^{\perp}$ or $\kappa_{\lambda}^{\|} / \kappa^{\|}$, depending on the transport direction. Therefore, we consider only those phonons that carry a significant fraction of the heat and neglect the relaxation times of those that do not contribute to the thermal conductivity. In this way, we estimate an upper bound of the time needed for the conductance to change from low to high and vice versa when the polarization is rotated, giving more weight to the modes that carry more heat. In Fig. 5, we plot (a) the relaxation times $\tau_{\lambda}$ as a function of the frequency and (b) the weighting factors $\kappa^{\perp, \|}(\nu) / \kappa^{\perp, \|}$, where we grouped the mode-by-mode contributions to $\boldsymbol{\kappa}$ in frequency intervals of $1 \mathrm{THz}$. We have obtained $\tau^{\perp}=7$ ps and $\tau^{\|}=5.8$ ps. These values are quite smaller than typical switching times of the polarization, and thus, their effect of the switching dynamics is negligible. Therefore, taking the

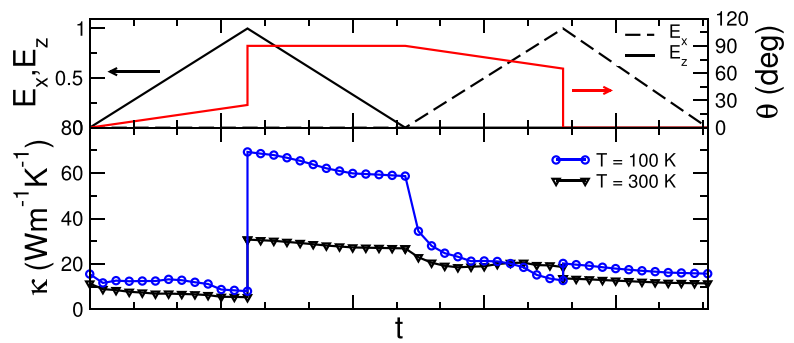

FIG. 4. Thermal conductivity as a function of time, $\boldsymbol{\kappa}(t)$, at $T=100$ and $300 \mathrm{~K}$ in response to the variation of the electric field, whose components $E_{x}$ and $E_{z}$ are plotted in the upper panel in units of the coercive field, $E_{c o e}$. The angle formed by the polarization vector $\theta$ and the direction of the heat flux is also shown. 


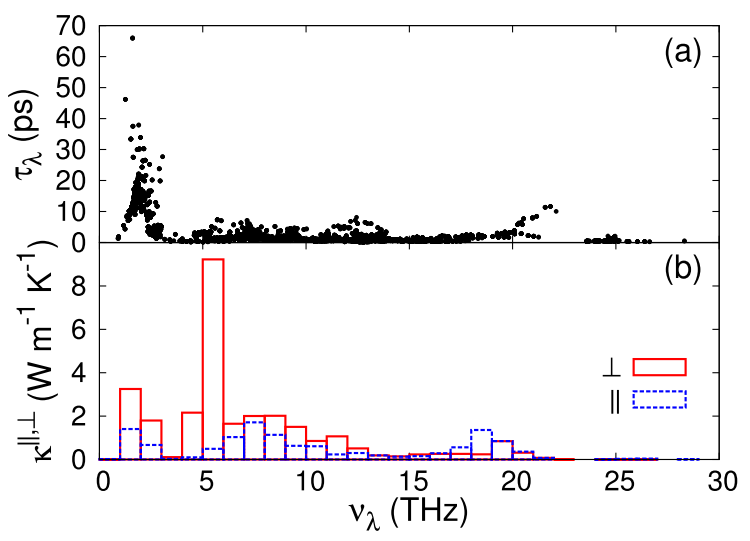

FIG. 5. (a) Relaxation time, $\tau_{\lambda}$, as a function of frequency. (b) Frequency resolved thermal conductivity, $\kappa^{\perp}(\nu)$ and $\kappa^{\|}(\nu)$.

ultrafast polarization switching time of 220 ps in thin-film ferroelectrics reported by $\mathrm{Li}$ and coworkers, ${ }^{22}$ we can estimate a maximum operation frequency of $4.5 \mathrm{GHz}$.

In conclusion, we present a proof-of-concept of a thermal switch that takes advantage of the anisotropy of the thermal conductivity in $\mathrm{PbTiO}_{3}$ and, thus, of the availability of built-in low and high conductivity states. At variance with common anisotropic materials, the electrically triggered rotation of the ferroelectric polarization can be used to switch between the two conductivity states. We also present a detailed study of the hysteresis cycle in the response of the thermal conductivity, showing that its value depends on how the electric field changed in the past. Finally, from the computed phonon relaxation times and contributions to the thermal conductivity, we argue that the response of such a ferroelectric thermal switch will be quite fast and only limited by the speed of the ferroelectric switch itself; hence, our proposed device should be able to operate in the high-frequency regime. The proposed scheme can be extended to any material (i) that has a high anisotropy of the thermal conductivity and (ii) whose anisotropy is directly related to a structural distortion that can be manipulated with an electric field.

We acknowledge financial support from the Ministerio de Economía, Industria y Competitividad (MINECO) under Grant No. FEDER-MAT2017-90024-P, the Severo Ochoa Centers of Excellence Program under Grant No. SEV-2015-0496, and the Generalitat de Catalunya under Grant No. 2017 SGR 1506. The work in Luxembourg was funded by the Luxembourg National Research Fund (Grant No. FNR/C18/MS/12705883/REFOX/ Gonzalez).

\section{REFERENCES}

${ }^{7}$ N. Li, J. Ren, L. Wang, G. Zhang, P. Hänggi, and B. Li, Rev. Mod. Phys. 84, 1045 (2012).

${ }^{2}$ S. Volz, J. Ordonez-Miranda, A. Shchepetov, M. Prunnila, J. Ahopelto, T. Pezeril, G. Vaudel, V. Gusev, P. Ruello, E. M. Weig, M. Schubert, M. Hettich, M. Grossman, T. Dekorsy, F. Alzina, B. Graczykowski, E. Chavez-Angel, J. Sebastian Reparaz, M. R. Wagner, C. M. Sotomayor-Torres, S. Xiong, S. Neogi, and D. Donadio, Eur. Phys. J. B 89, 15 (2016).

${ }^{3}$ I. Zardo and R. Rurali, Curr. Opin. Green Sustainable Chem. 17, 1 (2019).

${ }^{4}$ A. Togo, L. Chaput, and I. Tanaka, Phys. Rev. B 91, 094306 (2015).

${ }^{5}$ M. Raya-Moreno, H. Aramberri, J. A. Seijas-Bellido, X. Cartoixà, and R. Rurali, Appl. Phys. Lett. 111, 032107 (2017).

${ }^{6}$ M. Raya-Moreno, R. Rurali, and X. Cartoixà, Phys. Rev. Mater. 3, 084607 (2019).

${ }^{7}$ M. Harb, C. von Korff Schmising, H. Enquist, A. Jurgilaitis, I. Maximov, P. V. Shvets, A. N. Obraztsov, D. Khakhulin, M. Wulff, and J. Larsson, Appl. Phys. Lett. 101, 233108 (2012).

${ }^{8}$ J. A. Seijas-Bellido, H. Aramberri, J. Íniguez, and R. Rurali, Phys. Rev. B 97, 184306 (2018).

${ }^{9}$ P. Torres, J. A. Seijas-Bellido, C. Escorihuela-Sayalero, J. Íñiguez, and R. Rurali, Phys. Rev. Mater. 3, 044404 (2019).

${ }^{10}$ J. A. Seijas-Bellido, C. Escorihuela-Salayero, M. Royo, M. P. Ljungberg, J. C. Wojdet, J. Íñiguez, and R. Rurali, Phys. Rev. B 96, 140101 (2017).

${ }^{1}$ M. Royo, C. Escorihuela-Salayero, J. Íñiguez, and R. Rurali, Phys. Rev. Mater. 1, 051402 (2017).

${ }^{12}$ J. C. Wojdel, P. Hermet, M. P. Ljungberg, P. Ghosez, and J. Íñiguez, J. Phys.: Condens. Matter 25, 305401 (2013).

${ }^{13}$ P. García-Fernández, J. C. Wojdeł, J. Íñiguez, and J. Junquera, Phys. Rev. B 93, 195137 (2016).

${ }^{14}$ P. Zubko, J. C. Wojdeł, M. Hadjimichael, S. Fernandez-Pena, A. Sené, I. Luk'yanchuk, J. Triscone, and J. Íñiguez, Nature 534, 524 (2016).

${ }^{15}$ P. Shafer, P. García-Fernández, P. Aguado-Puente, A. R. Damodaran, A. K. Yadav, C. T. Nelson, S.-L. Hsu, J. C. Wojdeł, J. Íñiguez, L. W. Martin, E. Arenholz, J. Junquera, and R. Ramesh, Proc. Natl. Acad. Sci. U.S.A. 115, 915 (2018).

${ }^{16}$ A. Y. Serov, Z.-Y. Ong, and E. Pop, Appl. Phys. Lett. 102, 033104 (2013).

${ }^{17}$ M. Sledzinska, R. Quey, B. Mortazavi, B. Graczykowski, M. Placidi, D. Saleta Reig, D. Navarro-Urrios, F. Alzina, L. Colombo, S. Roche, and C. M. Sotomayor Torres, ACS Appl. Mater. Interfaces 9, 37905 (2017).

${ }^{18}$ W. Li, J. Carrete, N. A. Katcho, and N. Mingo, Comput. Phys. Commun. 185, 1747 (2014).

${ }^{19}$ W. Li, L. Lindsay, D. A. Broido, D. A. Stewart, and N. Mingo, Phys. Rev. B 86, 174307 (2012).

${ }^{20}$ P. Torres, A. Torelló, J. Bafaluy, J. Camacho, X. Cartoixà, and F. X. Alvarez, Phys. Rev. B 95, 165407 (2017).

${ }^{27}$ S. Tamura, Phys. Rev. B 27, 858 (1983).

${ }^{22}$ J. Li, B. Nagaraj, H. Liang, W. Cao, C. H. Lee, and R. Ramesh, Appl. Phys. Lett. 84, 1174 (2004). 